\title{
Spherical Colloids: Effect of Discrete Macroion Charge Distribution and Counterion Valence
}

\author{
René Messina* \\ Max-Planck-Institut für Polymerforschung, Ackermannweg 10, 55128 Mainz, \\ Germany
}

\begin{abstract}
We report the coupled effects of macroion charge discretization and counterion valence in the primitive model for spherical colloids. Instead of considering a uniformly charged surface, as it is traditionally done, we consider a more realistic situation where discrete monovalent microscopic charges are randomly distributed over the sphere. Monovalent or multivalent counterions ensure global electroneutrality. We use molecular dynamics simulations to study these effects at the ground state and for finite temperature. The ground state analysis concerns the counterion structure and charge inversion. Results are discussed in terms of simple analytical models. For finite temperature, strong and weak Coulomb couplings are treated. In this situation of finite temperature, we considered and discussed the phenomena of ionic pairing (pinning) and unpairing (unpinning).
\end{abstract}

Key words: Charged colloids, charge inversion, order-disorder transformations, molecular dynamics

PACS: 82.70.Dd, 61.20.Qg, 64.60.Cn

\section{Introduction}

Charged colloidal suspensions are a subject of intense experimental and theoretical work not only because of their direct application in industrial or biological processes, but also because they represent model systems for atomistic systems. The electrostatic interactions involved in such systems have a fundamental role in determining their physico-chemical properties [1,2]. Theoretical description of highly charged colloidal solutions faces two challenges: (i) different typical length scales due to the presence of macroions (i. e. charged colloids

\footnotetext{
* Tel: +496131379 148 Fax: +496131 379100

Email address: messina@mpip-mainz.mpg.de (René Messina).
} 
of the size 10-1000 $\AA$ ) and microscopic counterions and (ii) their long-range Coulomb interaction. A first simplifying assumption is to treat the solvent as a dielectric medium solely characterized by its relative permittivity $\epsilon_{r}$. A second widely used approximation consists in modeling the short range ion-ion excluded volume interaction by hard spheres. These two approximations are the basis of the so-called primitive model of electrolyte solutions. The system under consideration is an asymmetrical electrolyte solution made up of highly charged macroions and small counterions. A further simplification motivated by this asymmetry can be made by partitioning the system into subvolumes (cells), each containing one macroion together with its neutralizing counterions plus (if present) additional salt. This approximation has been called accordingly the cell model $[3,4]$. The cells adopt the symmetry of the macroion, here spherical, and are electrostatically decoupled. It is within the cell model that we present our simulation results.

For spherical macroions the structural charge is usually modeled by a central charge, which by Gauss theorem is equivalent to a uniform surface charge density as far as the electric field (or potential) outside the spherical colloid is concerned.

Most analytical concepts as well as simulations rely on the above assumptions and especially on the central charge assumption. It is well known that in the strong Coulomb coupling regime ion-ion correlations become very important, and significant deviations from mean-field approaches are expected. A counterintuitive effect which classical mean-field theories (like Poisson-Boltzmann model) cannot explain is the phenomenon of overcharge, also called charge inversion. That is, there are counterions in excess in the vicinity of the macroion surface so that its net charge changes sign. This has recently attracted significant attention [5-17]. In particular, we showed recently that this phenomenon may give rise to a strong long range attraction between like-sign charged colloids $[12,13,17]$. A natural question which comes up is: does overcharge and more generally ion-ion correlations strongly depend on the way the macroion structural charge is represented (i. e. uniformly charged or discrete charges on its surface)? In a recent paper [16], we studied such a situation in the strong Coulomb coupling regime where the macroion charge was carried by divalent microions in the presence of divalent counterions (same ionic valence). In Ref. [16] we reported the important result showing that overcharge is still possible under those conditions. Moreover we showed that the intrinsic electric field solely due to the macroion surface microions (without counterions) varies strongly from point to point on the colloidal sphere [16].

The goal of this paper is to study by means of molecular dynamics (MD) simulations the coupled effects of macroion charge discretization and counterion valence in the primitive model for spherical colloids. A systematic comparison with the uniform macroion charge distribution (i. e. central charge) is under- 
taken. The paper is organized as follows. In Sec. 2 we give some details on the macroion charge discretization as well as on the MD simulation model. Section 3 is devoted to the ground state analysis where surface counterion structure and overcharge are addressed. In Sec. 4 we investigate the finite temperature situation, where counterion structure is studied for strong and weak Coulomb couplings. Finally, in Sec. 5 we provide a summary of the results.

\section{Simulation model}

\subsection{Macroion charge discretization}

The procedure is similar to the one used in a previous study [16]. The discrete macroion charge distribution is produced by using $Z_{m}$ monovalent microions of diameter $\sigma$ (same diameter as the counterions) distributed randomly on the surface of the macroion. Then the structural charge is $Q=-Z_{m} e=-Z_{m} Z_{d} e$, where $Z_{m}>0, Z_{d}=1$ is the valence of these discrete microions and $e$ is the positive elementary charge. These discrete colloidal charges (DCC) are fixed on the surface of the spherical macroion. Figure 1 shows a schematic view of the setup. The counterions (not shown in Fig. 1) have a charge $q=+Z_{c} e$, where $Z_{c}>0$ stands for the counterion valence. In spherical coordinates the elementary surface is given by:

$$
d A=r_{0}^{2} \sin \theta d \theta d \varphi=-r_{0}^{2} d(\cos \theta) d \varphi
$$

where $r_{0}$ is the distance between the macroion center and the DCC center. Thus to produce a random discrete charge distribution on the surface we generated (uniformly) randomly the variables $\cos \theta$ and $\varphi$. Excluded volume is taken into account by rejecting configurations leading to an overlap of microions. Phenomena such as surface chemical reactions [18], hydration, roughness [19] are not considered. For commodity we introduce the notation $\left(-Z_{d}:+Z_{c}\right)$ to characterize the valence symmetry (asymmetry) for $Z_{c}=1\left(Z_{c}>1\right)$ of the ions (DCC and counterions) involved in discrete systems.

\subsection{Molecular dynamics procedure}

A MD simulation technique was used to compute the motion of the counterions coupled to a heat bath acting through a weak stochastic force $\mathbf{W}(\mathrm{t})$. The procedure is very similar to the one used in previous studies $[12,16]$. 


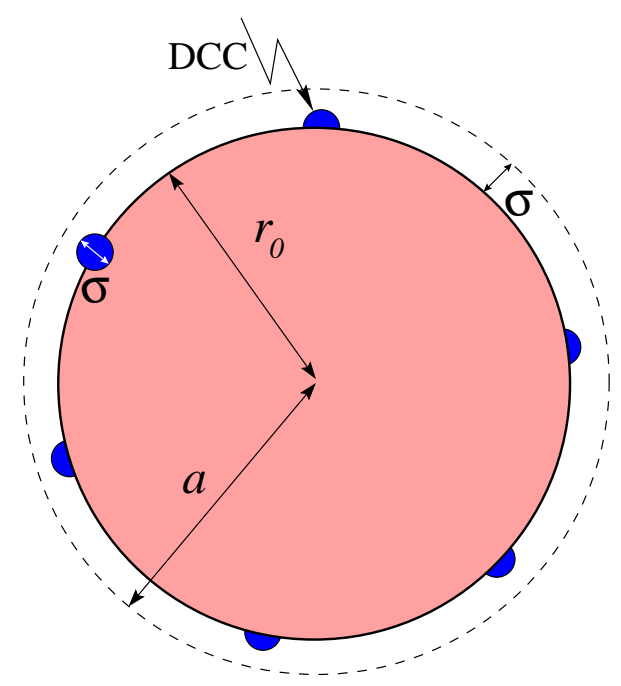

Fig. 1. Schematic view of the setup: the discrete colloidal charges (DCC) of diameter $\sigma$ are in dark grey. For a detailed meaning of the other symbols see text. Note that this a a two-dimensional representation of the three-dimensional system.

The motion of counterion $i$ (DCC ions being fixed) obeys the Langevin equation

$$
m \frac{d^{2} \mathbf{r}_{i}}{d t^{2}}=-\nabla_{i} U\left(\mathbf{r}_{i}\right)-m \gamma \frac{d \mathbf{r}_{i}}{d t}+\mathbf{W}_{i}(t)
$$

where $m$ is the counterion mass, $U$ is the potential force having two contributions: (i) the Coulomb interaction and (ii) the excluded volume interaction, and $\gamma$ is the friction coefficient. Friction and stochastic force are linked by the dissipation-fluctuation theorem $<\mathbf{W}_{i}(t) \cdot \mathbf{W}_{j}\left(t^{\prime}\right)>=6 m \gamma k_{B} T \delta_{i j} \delta\left(t-t^{\prime}\right)$. For the ground state simulations the stochastic force is set to zero.

Excluded volume interactions are taken into account with a pure repulsive Lennard-Jones potential given by

$$
U_{L J}(r)= \begin{cases}4 \epsilon_{L J}\left[\left(\frac{\sigma}{r-r_{0}}\right)^{12}-\left(\frac{\sigma}{r-r_{0}}\right)^{6}\right]+\epsilon_{L J}, & \text { for } r-r_{0}<r_{c u t} \\ 0, & \text { for } r-r_{0} \geq r_{c u t}\end{cases}
$$

where $r_{0}=0$ for the microion-microion interaction (the microion being either a counterion or a DCC), $r_{0}=7 \sigma$ for the macroion-counterion interaction and $r_{c u t}=2^{1 / 6} \sigma$ is the cutoff radius. This leads to a (center-center) macroioncounterion distance of closest approach $a=8 \sigma$ (see also Fig. 1). The macroion surface charge density $\sigma_{m}$ is defined as

$$
\sigma_{m}=\frac{Z_{m}}{4 \pi a^{2}}
$$


Table 1

Simulation parameters with some fixed values.

\begin{tabular}{ll}
\hline parameters & \\
\hline$\sigma=3.57 \AA$ & Lennard Jones length units \\
$T_{0}=298 \mathrm{~K}$ & room temperature \\
$\epsilon_{L J}=k_{B} T_{0}$ & Lennard Jones energy units \\
$Z_{m}$ & macroion valence \\
$Z_{d}=1$ & discrete colloidal charge valence \\
$Z_{c}$ & counterion valence \\
$l_{B}$ & Bjerrum length \\
$a=8 \sigma$ & macroion-counterion distance of closest approach \\
$\sigma_{m}$ & macroion surface charge density \\
$R=40 \sigma$ & simulation cell radius \\
$f_{m}=8 \times 10^{-3}$ & macroion volume fraction \\
\hline
\end{tabular}

Energy and length units in our simulations are related to experimental units by taking $\epsilon_{L J}=k_{B} T_{0}$ (with $T_{0}=298 \mathrm{~K}$ ) and $\sigma=3.57 \AA$ respectively.

The pair electrostatic interaction of any pair $i j$, where $i$ and $j$ denote either a DCC a counterion or the central charge (for the non-discrete case), reads

$$
U_{\text {coul }}(r)=k_{B} T_{0} l_{B} \frac{Z_{i} Z_{j}}{r}
$$

where $l_{B}=e^{2} / 4 \pi \epsilon_{0} \epsilon_{r} k_{B} T_{0}$ is the Bjerrum length describing the electrostatic strength. For the rest of this paper, electrostatic energy will always be expressed in units of $k_{B} T_{0}$. This also holds for the ground state analysis where the temperature is $T=0 \mathrm{~K}$ but $T_{0}=298 \mathrm{~K}$. From now on the pair electrostatic interaction will be written in reduced units so that Eq. (5) reads $U_{\text {coul }}=Z_{i} Z_{j} / r$.

The macroion and the counterions are confined in a spherical impenetrable cell of radius $R$. The macroion is held fixed and is located at the center of the cell. The colloid volume fraction $f_{m}$ is defined as $a^{3} / R^{3}$. To avoid image charge complications, the permittivity $\epsilon_{r}$ is supposed to be identical within the whole cell (including the macroion) as well as outside the cell. Typical simulation parameters are gathered in Table 1. 


\section{Ground state analysis}

In this section, we focus on counterion distribution exclusively governed by energy minimization, i. e. $T=0 \mathrm{~K}$. In such a case correlations are maximal and all the counterions lie on the macroion surface. This situation has the advantage to enable accurate computation of energy variations in processes such as overcharging and also to provide a clear description of effects which are purely correlational in nature. The method employed here was successfully carried out in Refs. $[12,13,16,17]$ and is explained in details in Ref. [17]. The Bjerrum length $l_{B}$ is set to $10 \sigma$. Note that in the ground state the value of $l_{B}$, or equivalently the value of the dielectric constant $\epsilon_{r}$, does not influence at all the counterion structure. Only the electrostatic energy is rescaled accordingly.

\subsection{Neutral case}

First we consider the simple case where the system [macroion + counterions] is globally neutral. In order to characterize the two-dimensional counterion structure we compute the counterion correlation function $(\mathrm{CCF}) g_{c}(r)$ on the surface of the sphere defined as

$$
c^{2} g_{c}\left(r=\left|r^{\prime}-r^{\prime \prime}\right|\right)=\sum_{i \neq j} \delta\left(r^{\prime}-r_{i}\right) \delta\left(r^{\prime \prime}-r_{j}\right)
$$

where $c=N_{c} / 4 \pi a^{2}$ is the surface counterion concentration $\left(N_{c}=Z_{m} / Z_{c}\right.$ being the number of counterions) and $r$ corresponds to the arc length on the sphere. Note that at zero temperature all equilibrium configurations are identical (except for degenerate ground state), thus only one is required to obtain $g_{c}(r)$. The counterion correlation function $g_{c}(r)$ is normalized as follows

$$
c \int_{0}^{\pi a} 2 \pi r g_{c}(r) d r=\left(N_{c}-1\right) .
$$

Because of the finite size and the topology of the sphere, $g(r)$ has a cut-off at $r_{g c}=\pi a=25.1 \sigma$ and $g\left(r_{g c}\right)=0$. Furthermore the absolute value of $g(r)$ can not be directly compared to the one obtained with an infinite plane.

Similarly, one can also define a surface macroion correlation function (MCF) $g_{m}(r)$ for the microions (representing the colloidal structural charge) on the surface of the macroion. The normalization of $g_{m}(r)$ is very similar to Eq. (7) 
and reads

$$
\sigma_{m} \int_{0}^{\pi a} 2 \pi r g_{m}(r) d r=\left(Z_{m}-1\right)
$$

where the arc length has been rescaled by a factor $a / r_{0}$ so that $g_{c}(r)$ and $g_{m}(r)$ are directly comparable (see also the setup Fig. 1) and are defined in the same $r$ range.

\subsubsection{Monovalent counterions}

We first treat the systems where we have monovalent counterions, that is we have to deal with the symmetric discrete system $(-1:+1)$. The counterion correlation functions $g_{c}(r)$ are computed for a central macroion charge [denoted by $g_{c}^{(\mathrm{CC})}(r)$ ] and for discrete macroion charge distribution [denoted by $g_{c}^{(\mathrm{DCC})}(r)$ ]. Results for three structural charges $Z_{m}=60,180$ and 360 are given in Figs. 2(a), (b) and (c) respectively. For the continuous case (central charge) the counterion structure consists of a pseudo-Wigner crystal (WC) as was already found in Refs. $[12,13,16,17]$. Also the higher the absolute number of counterions $N_{c}$ (i. e. the concentration $c$ ) the higher the order of counterion structure for the continuous case [compare Fig. 2(a) with Fig. 2(c)].

It turns out that in the case of discrete colloidal charges the counterion distribution is strongly dictated by the colloidal charge distribution and especially for low macroion surface charge density $\sigma_{m}\left(Z_{m}=60\right)$ [see Fig. 2(a)]. For $Z_{m}=60, g_{c}^{(\mathrm{DCC})}(r)$ and $g_{m}(r)$ are almost identical. This indicates that each counterion is exactly associated with one DCC site. The ground state structure for $Z_{m}=60$ is depicted in Fig. 3(a) where one clearly observes this ionic pairing.

This strong ionic pair association can be easily explained in terms of local correlations. Let us consider the picture sketched in Fig. 4 which holds for strong ionic pairing, where a given dipole A (ionic pair made up of a counterion and a DCC site) on the macroion surface essentially interacts with its first nearest surrounding dipoles B. Note that very similar lengths were also considered in a recent theoretical study [20] in the one-dimensional case (counterion adsorption on a linear polyelectrolyte). It is important to have in mind that such a local description is physically justified due to the strong screening generated by ionic pairing. Thereby local correlations are twofold: (i) the attractive interaction between the DCC site of dipole A with its paired counterion and the counterions of dipoles $\mathrm{B}$, and (ii) the repulsive interaction between the counterion of dipole $\mathrm{A}$ and counterions of dipoles $\mathrm{B}$. The correlations between DCC sites are not relevant since they are fixed. The intra-dipole attractive 
(a)

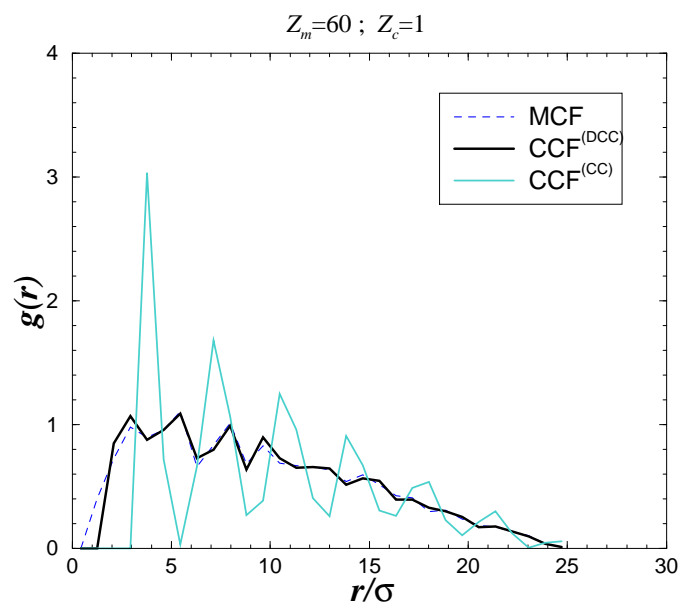

(b)

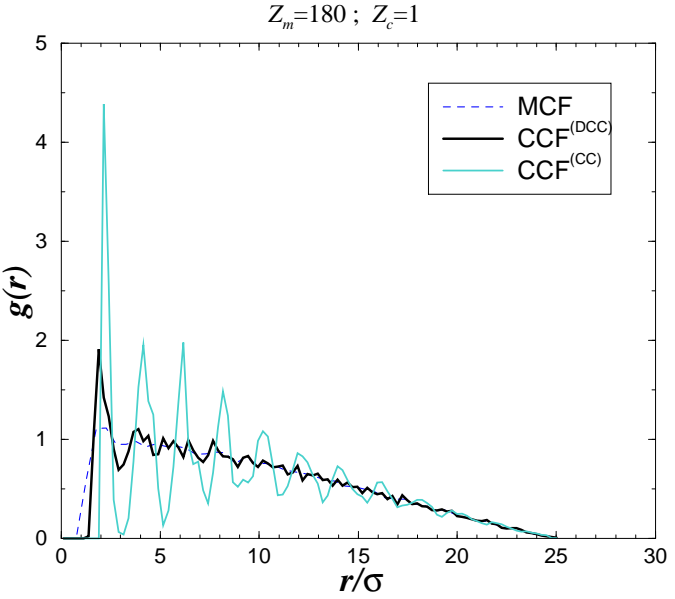

(c)

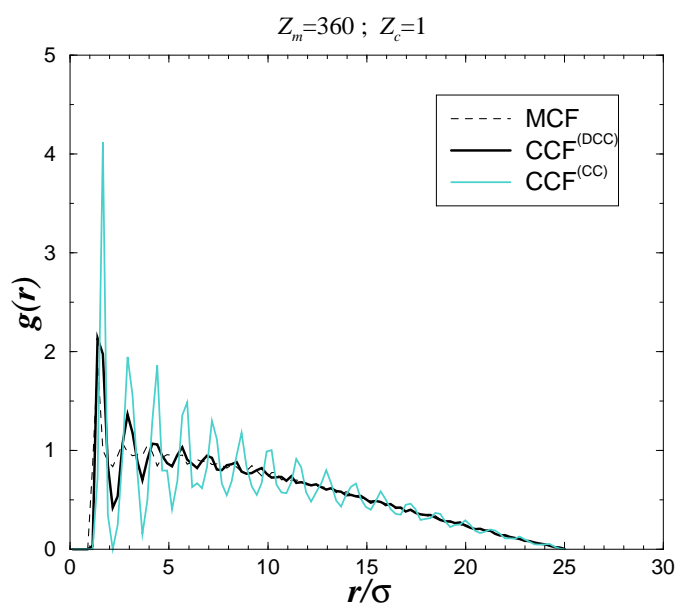

Fig. 2. Ground state surface correlation functions $g(r)$ for monovalent counterions $\left(Z_{c}=1\right)$ and for three macroion bare charges: (a) $Z_{m}=60$ (b) $Z_{m}=180$ and (c) $Z_{m}=360$. The two counterion correlation functions $(\mathrm{CCF}) g_{c}(r)$ are obtained for discrete colloidal charges $\left[g_{c}^{(\mathrm{DCC})}(r)\right.$ denoted by $\left.\mathrm{CCF}^{(\mathrm{DCC})}\right]$ and for the central charge $\left[g_{c}^{(\mathrm{CC})}(r)\right.$ denoted by $\left.\mathrm{CCF}^{(\mathrm{CC})}\right]$. MCF stands for the discrete colloidal charges pair distribution $g_{m}(r)$.

interaction $E_{\text {pin }}$ between the DCC site and its "pinned" counterion can be written as

$$
E_{p i n}=-\frac{Z_{d} Z_{c}}{\sigma}
$$

For the elementary nearest inter-dipole (or inter-ionic pair) interactions, one can write for the attractive interaction $E_{+-}$between the DCC site of dipole $\mathrm{A}$ and the counterion of dipole B:

$$
E_{+-}=-\frac{Z_{d} Z_{c}}{a_{d c}}
$$



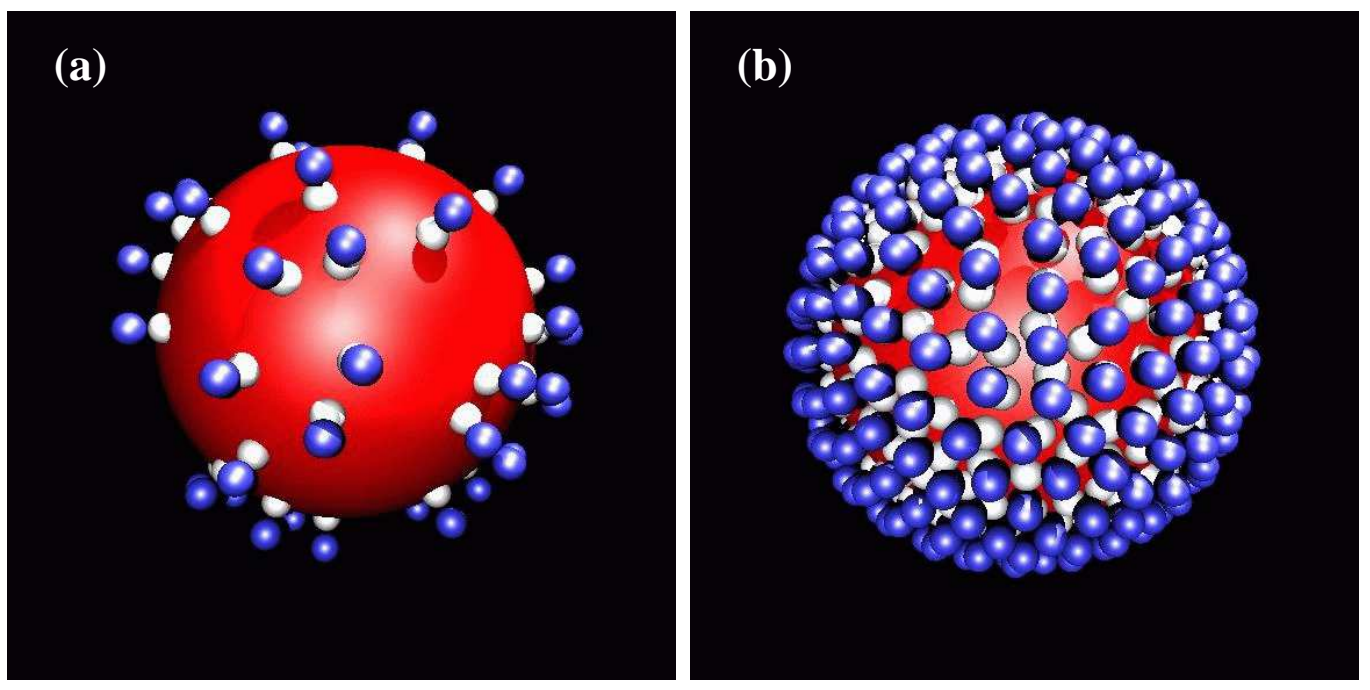

Fig. 3. Ground state structures for discrete monovalent systems $(-1:+1)$ : (a) $Z_{m}=60$ and (b) $Z_{m}=360$. The discrete colloidal charges (DCC) are in white, and the counterions in blue. Full ionic pairing association occurs. The corresponding counterion correlation functions $g_{c}(r)$ can be found in Figs. 2(a) and (c).

A similar expression can be written for the repulsive inter-dipole interaction $E_{++}$involving counterions of dipole $\mathrm{A}$ and dipole B, which reads

$$
E_{++}=\frac{Z_{c}^{2}}{a_{c c}} .
$$

Note that the repulsive counterion-counterion term $E_{++}$alone, even if space truncated $\square$, drives to the long-range ordered triangular WC structure. However at zero temperature the DCC sites represent pinning centers for the counterions where the electrostatic potential is considerably lowered (due to the

$\overline{1}$ This statement holds if the cutoff is larger than the lattice constant.

\section{dipole A dipole B}

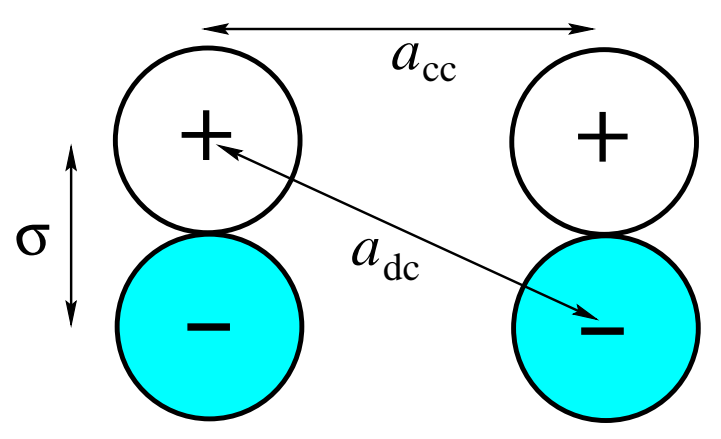

Fig. 4. Schematic view of the local electrostatic interactions and typical correlation lengths involved between nearest dipoles. The negatively charged DCC (-) are in grey and the positively charged counterions $(+)$ in white. 
term $E_{\text {pin }}$ ) compared to its direct "empty" neighborhood (charge hole), which in turn prevents the counterions from adopting the ideal WC structure. This latter aspect was thoroughly discussed in Ref. [16]. Another important quantity characterizing discrete systems is the ratio

$$
\rho_{p i n}=\frac{\widetilde{a}_{c c}}{d_{p i n}}
$$

between the mean inter-dipole separation $\widetilde{a}_{c c}$ (more exactly the mean counterioncounterion separation) and intra-dipole separation $d_{p i n}$ of an ionic pair (in the present study $d_{p i n}=\sigma$ as depicted in Fig. 4). The value of $\widetilde{a}_{c c}$ can be obtained by taking the first peak position of $g_{c}^{(\mathrm{CC})}(r)$.

Obviously, for sufficiently low macroion surface charge density $\sigma_{m}$ (i. e. large $\left.\rho_{\text {pin }}\right)$ the ionic pairing term $E_{\text {pin }}$ will be dominant and strong ionic pairing occurs. More specifically, when the typical inter-dipole distance is large compared to the intra-dipole distance then dipole-dipole interactions are weak (i. e., $\left.\left|E_{+-}-E_{++}\right| \ll\left|E_{\text {pin }}\right|\right)$ and the DCC distribution dictates the counterion structure. This is what qualitatively explains our simulation findings for $Z_{m}=60$ [see Fig. 2(a) and Fig. 3(a)].

When $\sigma_{m}$ becomes sufficiently important the situation may become qualitatively different. In this case dipoles approach each other and because of excluded volume ${ }^{2} a_{c c}$ becomes comparable to $\sigma$ (see Fig. 4) ${ }^{3}$. Thereby, the counterion-counterion repulsion term $E_{++}$(overcompensating $E_{+-}$) induces counterion ordering compatible with the local attractive pinning potential field generated by DCC centers. This effect can be inspected in Fig. 2(b) and Fig. $2(\mathrm{c})$ where one sees that upon increasing $\sigma_{m}, g_{c}^{(\mathrm{DCC})}(r)$ is gradually less correlated with $g_{m}(r)$ and more correlated with $g_{c}^{(\mathrm{CC})}(r)$. As a topological consequence, some counterions will be in contact with several (two or more) DCC attractors as can be seen in Fig. 3(b).

The quasi-triangular counterion arrangement for high $\sigma_{m}\left(Z_{m}=360\right)$ can be inspected in Fig. 3(b). For this symmetric system in size (same diameter for the counterions and the DCC ions) one expects that for a compact amorphous DCC layer the counterion structure should become perfectly ordered. This extreme limit which would correspond to unreachable experimental charge densities has not been addressed in our simulations.

In parallel, increasing $\sigma_{m}$ induces by purely excluded volume effect a stronger local order within the DCC layer as can be checked on the $g_{m}(r)$ plots in Figs.

2 Note that in the present model no surface dipole flip is allowed which should also be the case experimentally.

3 The limiting case is where the global structure is compact, i. e. touching spherical microions. 
2(a)-(c). This is quite similar to what occurs in a system of hard spheres where the (dense) liquid phase is locally correlated and the (dilute) gaseous phase is uncorrelated.

In summary, the system depicted above is the siege of an order-disorder phase transition where upon increasing $\sigma_{m}$ (i. e. decreasing $\rho_{\text {pin }}$ ) we pass from a disordered counterion structure (imposed by the DCC layer) to a long-range ordered one which is induced by local counterion-counterion correlations.

Although results presented above concern one given random distribution (for each $Z_{m}$ ), we carefully checked that similar results and conclusions could be drawn for different random realizations (systematically five). This also holds for the following section below where we deal with multivalent counterions.

\subsubsection{Multivalent counterions}

We turn to the asymmetric discrete systems $\left(-1:+Z_{c}\right)$ where multivalent counterions are present $\left(Z_{c}>1\right)$. The correlation functions $g(r)$ for two macroion charges $Z_{m}=60$ and $Z_{m}=180$ and various counterion valences $Z_{c}$ can be found in Fig. 5. One remarks that upon decreasing the number of counterions $N_{c}$ (i.e., increasing $Z_{c}$ ) for fixed $Z_{m}$, the first peak of $g_{c}(r)$ is gradually shifted to the right (compare also the monovalent case given in Fig. 2) whatever the nature of the macroion charge is (discrete or continuous). Furthermore, we observe for the discrete systems that upon increasing $Z_{c}$ (for fixed $\left.Z_{m}\right)$ the correlation between $g_{c}^{(\mathrm{DCC})}(r)$ and $g_{m}(r)$ decreases and increases between $g_{c}^{(\mathrm{DCC})}(r)$ and $g_{c}^{(\mathrm{CC})}(r)$. This effect is clearly noticeable in Fig. 2(b) and Figs. 5(c-e) corresponding to $Z_{m}=180$. The very high counterion valence $Z_{c}=10$ reported in Fig. 5(e) was undertaken in order to stress the counterion multivalence effect. These findings lead to the conclusion that the counterion valence has the effect of reducing the disorder in the counterion structure stemming from the randomness of the DCC distribution.

This related phenomenon can be theoretically explained with simple ideas. Basically, the mechanisms involved in this counterion valence induced ordering stem from two concomitant sources: (i) topological and (ii) correlational.

The topological aspect is due to the presence of $\left(Z_{m}-Z_{m} / Z_{c}\right)$ unbound DCC sites (free of associated counterion) ensuring global electroneutrality [compare for instance Fig. 3(a) and Fig. 6]. It is to say that here, compared to the monovalent case $(-1:+1)$, the counterions have all the more "freedom" to choose their pinning locations because $Z_{c}$ is high. To be more precise, the 
(a)

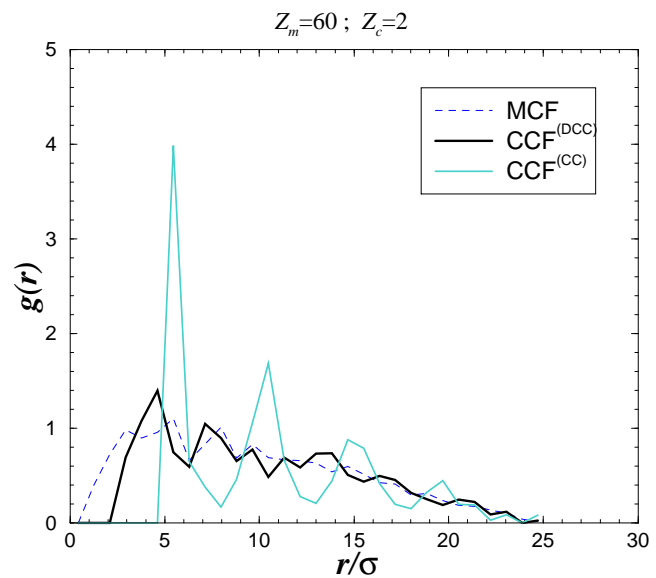

(c)

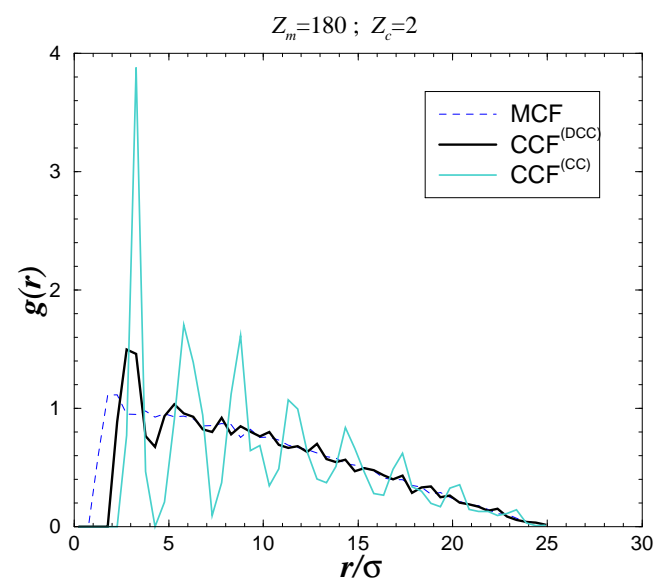

(b)

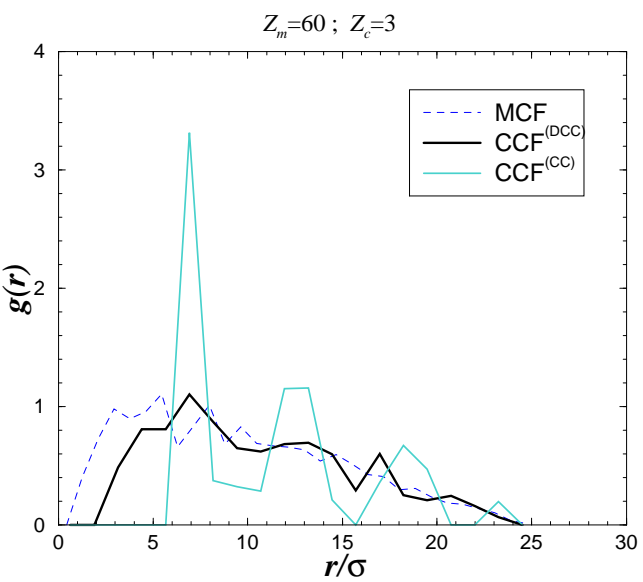

(d)

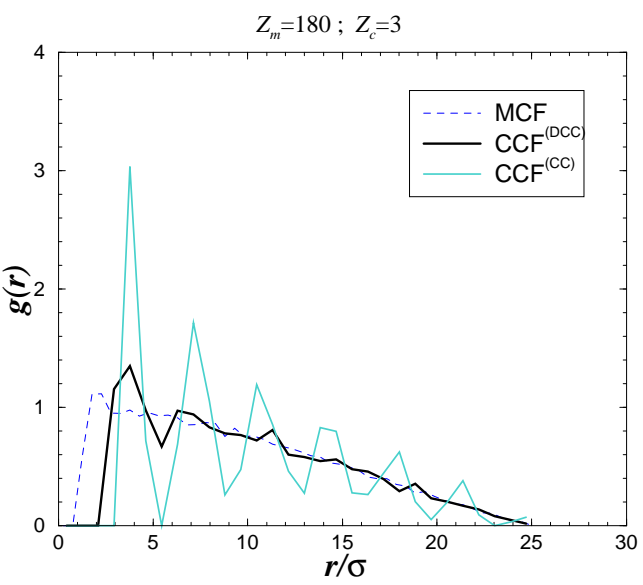

(e)

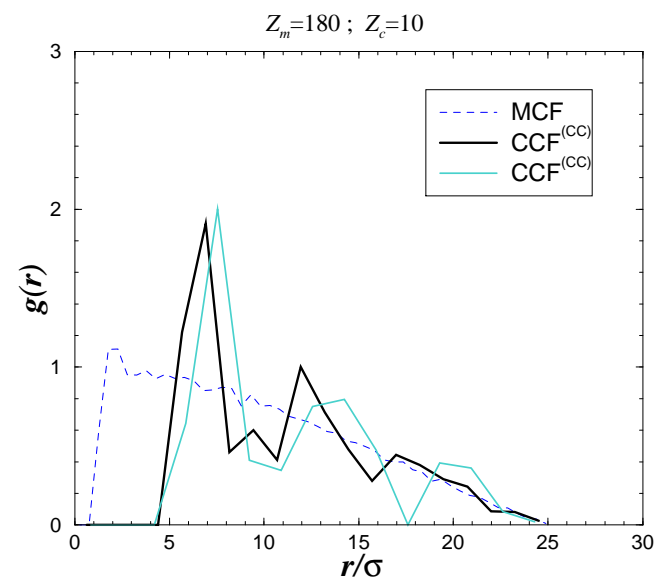

Fig. 5. Ground state surface correlation functions for different multivalent systems: (a) $Z_{m}=60, Z_{c}=2$; (b) $Z_{m}=60, Z_{c}=3$; (c) $Z_{m}=180, Z_{c}=2$; (d) $Z_{m}=180$, $Z_{c}=3$; (e) $Z_{m}=180, Z_{c}=10$. The two counterion correlation functions (CCF) are obtained for discrete colloidal charges (DCC) and for the central charge (CC). The $g_{m}(r)$ curves (denoted by MCF) are identical from (a) to (b) and from (c) to (e). 


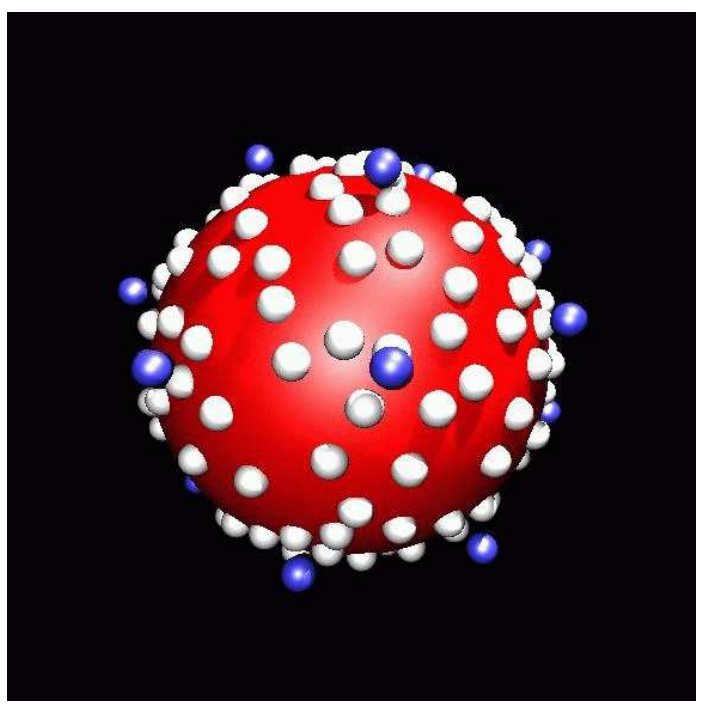

Fig. 6. Ground state structure for $(-1:+10)$ with $Z_{m}=180$. The corresponding counterion correlation function $g_{c}(r)$ can be found in Figs. 5(e).

number of topologically accessible "pinned" configurations is given 4 by

$$
C_{Z_{m}}^{\frac{Z_{m}}{Z_{c}}}=\frac{Z_{m} !}{\left(Z_{m}-\frac{Z_{m}}{Z_{c}}\right) !\left(\frac{Z_{m}}{Z_{c}}\right) !}
$$

which reduces to 1 for $Z_{c}=1$. In the ground state, counterions will "decide" to choose among these various possible arrangements the one which minimizes the total energy of the system. It is clear that this topological feature by itself promotes counterion valence induced ordering.

Concomitantly, there is a purely counterion correlation induced ordering which is $Z_{c}$ dependent. Indeed, using similar arguments as those previously employed for monovalent systems $(-1:+1)$ built on Eqs. (9-11), one can infer the role of $Z_{c}$. More specifically, by assuming an ordered WC structure' the term $E_{++}$ given by Eq. (11) can be rewritten as

$$
E_{++}^{W C} \sim \frac{Z_{c}^{3 / 2} Z_{m}^{1 / 2}}{a}
$$

4 Rigorously, Eq. (13) holds when each counterion is associated with one and only one DCC site (case of low $\sigma_{m}$ ). For high $\sigma_{m}$, it remains a good approximation to capture the essential physics.

5 From a topological point of view, it consists in replacing the current (random) Voronoi structure by the ordered WC structure. 
where $a_{c c}$ in Eq. (11) is now given by

$$
a_{c c}=\widetilde{a}_{c c} \sim c^{-1 / 2} \sim\left(\frac{Z_{m}}{Z_{c} a^{2}}\right)^{-1 / 2} .
$$

Equation (14) shows that for fixed $Z_{m}$ and $a$ (i. e., fixed macroion charge density) $E_{++}^{W C} \sim Z_{c}^{3 / 2}$ whereas $E_{\text {pin }} \sim Z_{c}$ (recalling that $Z_{d}=1$ ) and therefore for sufficiently high $Z_{c}$ the term $E_{++}^{W C}$ will be dominant. Thereby $Z_{c}$ induces counterion ordering so as to minimize mutual counterion-counterion repulsion merely dictated by Eq. (14). As a topological consequence, some counterions which would be in contact with several DCC sites if they were monovalent can now be in contact with less DCC sites (see Fig. 6).

In summary, these discrete multivalent systems are again the siege of an orderdisorder phase transition which is counterion valence controlled.

\subsection{Overcharge}

We now investigate the charge inversion (overcharge) phenomenon. The starting equilibrium configurations correspond to neutral ground states as were previously obtained. The method employed here is very similar to the one used in Refs. [12,16]. To produce a controlled overcharge, one adds successively overcharging counterions (OC) in the vicinity of the macroion surface. Thereby the resulting system is no longer neutral. Using Wigner crystal concepts $[6,21]$, we showed that the gain in electrostatic energy (compared to the neutral state) by overcharging a single uniformly charged macroion (i.e., central charge) with $n$ overcharging counterions can be written in the following way $[12,13,17]$ :

$$
\Delta E_{n}^{O C}=\Delta E_{n}^{c o r}+\Delta E_{n}^{m o n}=-\frac{\alpha Z_{c}^{2}}{\sqrt{A}}\left[\left(N_{c}+n\right)^{3 / 2}-N_{c}^{3 / 2}\right]+Z_{c}^{2} \frac{n^{2}}{2 a} .
$$

As before $N_{c}=Z_{m} / Z_{c}$ is the number of counterions in the neutral state, $A$ is the macroion area $\left(4 \pi a^{2}\right)$ and $\alpha$ is a positive constant which was determined by using simulation data for $\Delta E_{1}^{O C} . \Delta E_{n}^{c o r}$, which is equal to the first term of the right member, denotes the gain in energy due to ionic correlations. The derivation of this term can be found in Refs. [12,13,17], and the basic idea is that each counterion interacts essentially with its neutralizing uniformly charged Wigner-Seitz cell. The second term on the right hand side, $\Delta E_{n}^{\text {mon }}$, is the self-energy of the excess of charge. This repulsive term stops the overcharging for sufficiently large $n$. Note that the WC concept for describing energy correlations is already excellent for highly short range ordered structures (strongly correlated liquids, see Ref. [6] for a detailed discussion). The 
total electrostatic energy of the system as a function of $n$ is displayed Fig. 7 (for monovalent counterions) and Fig. 8 (for multivalent counterions) for two bare charges $Z_{m}=60$ and $Z_{m}=180$. The energy curves corresponding to discrete systems were produced by systematically averaging over five random DCC realizations.

\subsubsection{Monovalent counterions}

Let us first focus on the monovalent symmetric case $(-1:+1)$ where for the neutral state each DCC site is exactly associated with one counterion as was shown above. The results in Figs. 7(a-b) show that the overcharging process occurring with a discrete macroion charge distribution is quite different from the one obtained with an uniform surface charge distribution. Especially for the smallest bare charge $Z_{m}=60$, the effect of disorder is very important in agreement with what was already found above for the neutral state in Sec. 3.1.1. The main effects of charge discretization are: (i) the reduction in gain of energy and (ii) the reduction of maximal (critical) number, $n^{*}$, of stabilizing overcharging counterions (corresponding to a minimum in the energy curve). Both points were thoroughly discussed elsewhere [16] for an equivalent symmetric discrete system $(-2:+2)$. It was shown that points (i) and (ii) can be explained in terms of ion-dipole interaction, which presently is the main driving force for overcharging. When the overcharging counterions are present, each of them will essentially interact (attractively) with its neighboring dipoles (ionic pairs). The attractive ion-dipole interaction increases with decreasing ion-dipole separation, i. e. increasing macroion charge density $\sigma_{m}$. This explains why the energy gain increases with $Z_{m}$ [compare Fig. $7(\mathrm{a})$ and Fig. 7(b)]. On the other hand, the repulsion between the counterions is not fully minimized since they do not adopt the ideal WC structure that is obtained with a central charge which in turn explains (i) and (ii). However for high bare charge $\left(Z_{m}=180\right)$ the overcharge curve obtained with DCC [see Fig. 7(b)] approaches the one from the continuous case as expected for high counterion concentration. This feature is fully consistent with what was already found in Sec. 3.1.1, where it was shown that the order of the counterion structure in the neutral state (for discrete systems) increases with $\sigma_{m}$. In other terms, the WC approach through Eq. (16) is a good approximation for describing discrete systems at high $\sigma_{m}$ since stronger ordering exists.

Common features of overcharging between continuous and discrete systems are briefly given here. We note that $n^{*}$ increases with the macroionic charge $Z_{m}$. Furthermore, for a given $n$, the gain in energy always increases with $Z_{m}$. Also, for a given macroionic charge $Z_{m}$, the gain in energy between two successive overcharged states is decreasing with $n$. Note that at $T=0 \mathrm{~K}$, the value of $\epsilon_{r}$ acts only as a prefactor. All these features are captured by Eq. (16). 
(a)

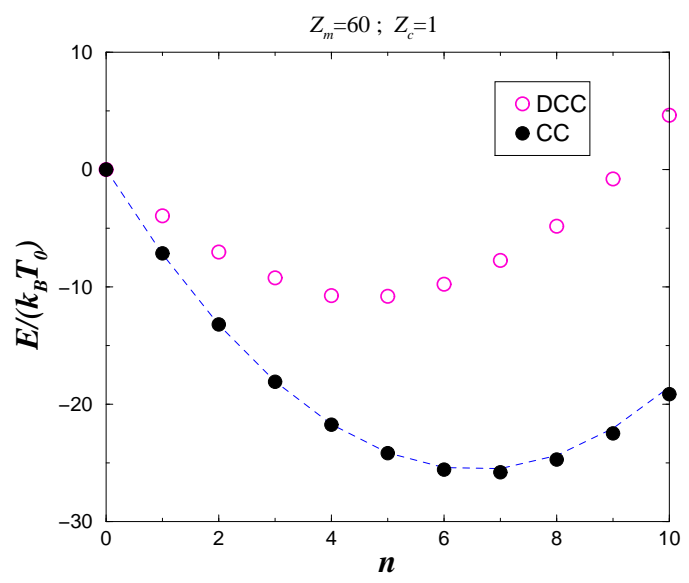

(b)

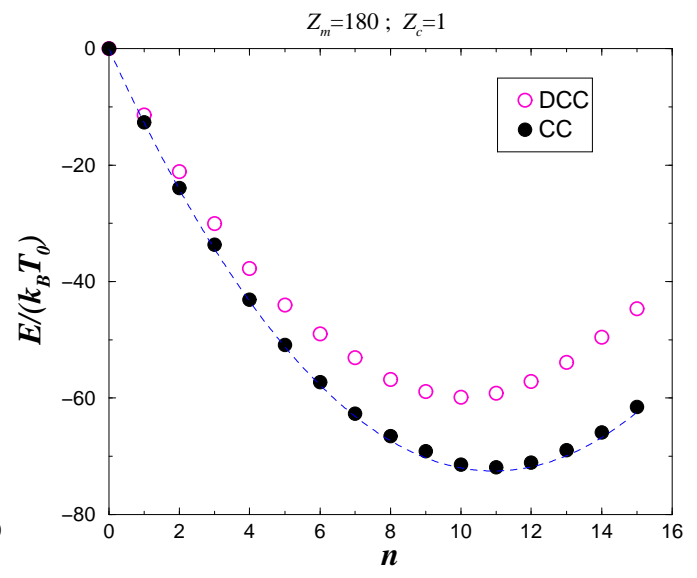

Fig. 7. Total electrostatic energy for monovalent counterions ground state configurations as a function of the number of overcharging counterions $n$ : (a) $Z_{m}=60$ (b) $Z_{m}=180$. Overcharge curves were computed for discrete macroion charge distribution (DCC) and macroion central charge (CC). The neutral case was chosen as the potential energy origin. Dashed lines were produced by using Eq. (16). For discrete systems (DCC) error bars are smaller than symbols.

\subsubsection{Multivalent counterions}

Now we are going to discuss the asymmetric discrete systems $\left(-1:+Z_{c}\right)$ where multivalent counterions are present $\left(Z_{c}>1\right)$. The results of figures 8 (a-d) indicate that the energy gain in the overcharging process at fixed $Z_{m}$ and $n$ is higher the higher the counterion valence $Z_{c}$ for both macroion charge distributions (discrete and continuous). For the continuous case this can be directly explained in terms of WC concepts [i. e. Eq. (16)]. Indeed the main leading term of the correlational energy $\Delta E_{n}^{c o r}$ in Eq. (16) scales like

$$
\Delta E_{n}^{c o r} \sim-Z_{c}^{3 / 2}
$$

for fixed $n$ and fixed macroion charge $Z_{m}$, and recalling that $N_{c}=Z_{m} / Z_{c}$. Equation (17) quantitatively (qualitatively) explains why overcharging is stronger with increasing counterion valence $Z_{c}$ for the continuous (discrete) case.

As far as discrete systems are concerned, the overcharging mechanisms occurring with multivalent counterions differ from those occurring with symmetric monovalent systems $(-1:+1)$. This is again due to the presence of $\left(Z_{m}-Z_{m} / Z_{c}\right)$ unbound DCC sites in the neutral state as discussed in Sec. 3.1.2. When overcharging comes into play, each overcharging counterion becomes paired with some ${ }^{\circ}$ of these free DCC sites. Figure 8 shows that the overcharging with

$\overline{6}$ It can be one or more depending on the valence, surface charged density and the local DCC site arrangement. 
(a)

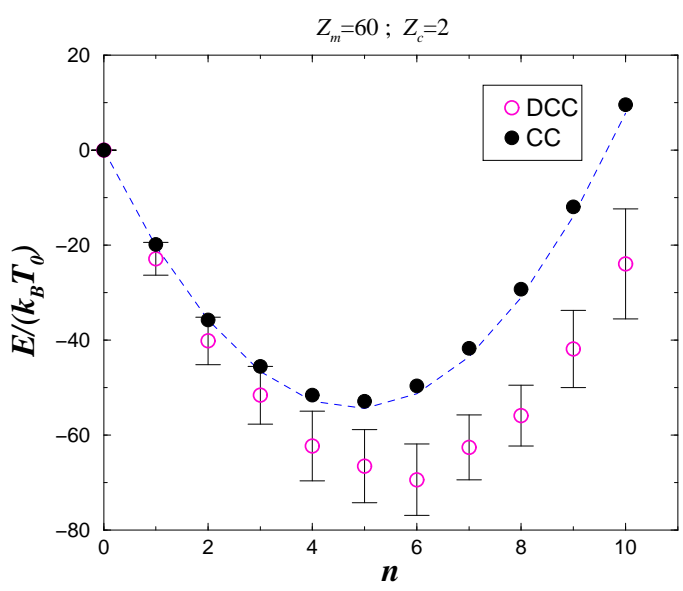

(c)

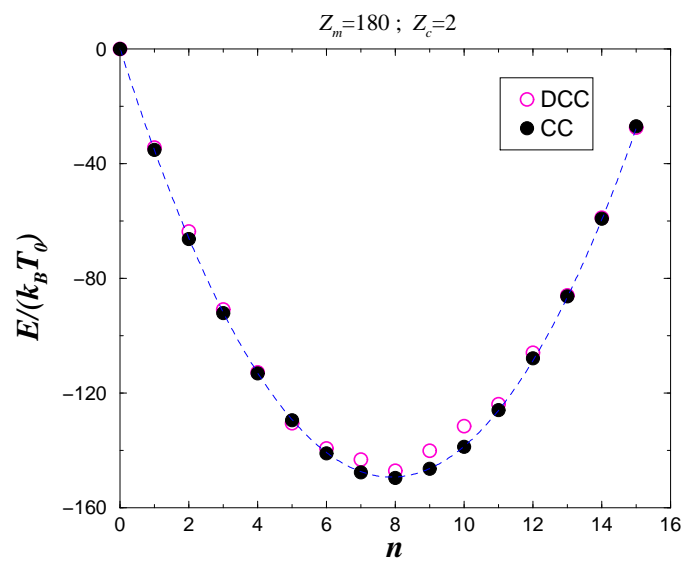

(b)

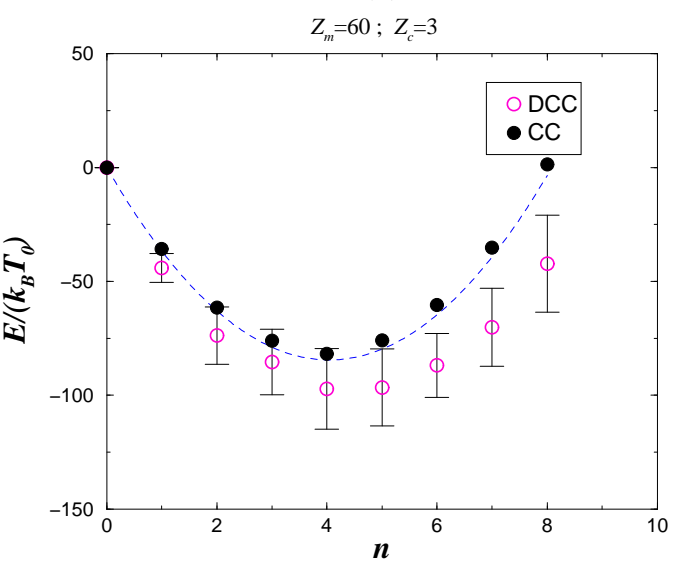

(d)

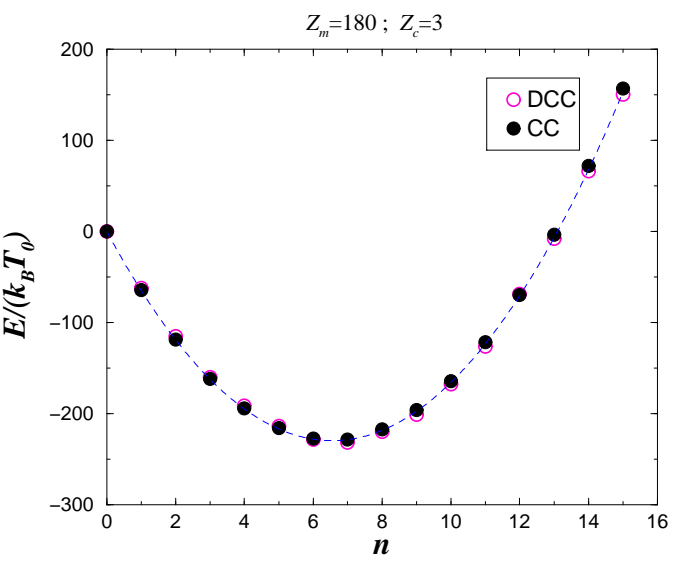

Fig. 8. Total electrostatic energy for multivalent counterions ground state configurations as a function of the number of overcharging counterions $n$ : (a) $Z_{m}=60$, $Z_{c}=2$ (b) $Z_{m}=60, Z_{c}=3$ (c) $Z_{m}=180, Z_{c}=2$ (d) $Z_{m}=180, Z_{c}=3$. Overcharge curves were computed for discrete macroion charge distribution (DCC) and macroion central charge (CC). The neutral case was chosen as the potential energy origin. Dashed lines were produced by using Eq. (16). For discrete systems (DCC) error bars are only indicated when larger than symbols.

multivalent counterions (especially the higher $Z_{m}$ ) is significantly less affected by colloidal charge discretization than in the monovalent case (see Fig. 7).

For $Z_{m}=60$, simulations show that overcharging in the discrete case can even be stronger than in the continuous case [see Figs. 8(a) and (b)]. This phenomenon can be qualitatively understood by referring to the very low macroion surface charge density limit, where the correlation term $\Delta E_{n}^{c o r}$ in Eq. (16) becomes negligible compared to the ionic pairing term $E_{\text {pin }}$ given by Eq. (9). In this limiting situation, the energy gain by overcharging is approximatively given by $-n Z_{d} Z_{c} / d_{\text {pin }}$ so that full overcharging occurs where each monovalent DCC site is paired with one multivalent counterion. 
For $Z_{m}=180$, the overcharging curves for discrete and continuous distributions are almost identical [see Figs. 8(c-d)]. This is consistent with what we already found in Sec. 3.1.2 for the counterion structure in the neutral state, where we showed that $g_{c}^{(\mathrm{DCC})}(r)$ approaches $g_{c}^{(\mathrm{CC})}(r)$ with increasing $Z_{c}$. However, the agreement between discrete and continuous cases is even better for overcharging than for counterion structure [see the corresponding $g_{c}(r)$ given in Figs. 5(c) and (d)]. This is due to the fact that, as previously mentioned, the WC approach [Eq. (16)] quantifying the energy gain by overcharging is already excellent for highly short-ranged ordered systems. Generally speaking, all the ordering mechanisms related in Sec. 3.1 for neutral discrete systems hold for the overcharging features: all causes leading to ordering enhance overcharging.

\section{Finite temperature}

In this part, the system is globally neutral and is brought to room temperature $T_{0}$. We are interested in determining the counterion distribution as well as the counterion motion within the counterion layer. The cell radius $R$ is fixed to $40 \sigma$ so that the macroion volume fraction $f_{m}$ has the finite value $8 \times 10^{-3}$.

\subsection{Strong Coulomb coupling}

The Bjerrum length $l_{B}$ is set to $10 \sigma$ as previously in the ground state study Sec. 3.1. In this section we consider two macroion bare charges $Z_{m}$ (60 and 180) and three counterion valences $Z_{c}$ (1,2 and 3). A typical parameter for describing the Coulomb coupling strength is the so-called plasma parameter $\Gamma$ [22] defined as $\Gamma=l_{B} Z_{c}^{2} / \widetilde{a}_{c c}$. For our simulation parameters, $\Gamma$ ranges from 2.6 (for $Z_{m}=60$ and $Z_{c}=1$ ) up to 23.1 (for $Z_{m}=180$ and $Z_{c}=3$ ). Under these conditions, systems are still highly energy dominated so that at equilibrium almost all (if not all depending on $Z_{m}$ and $Z_{c}$ ) counterions lie in the vicinity of the macroion surface (strong condensation). Therefore for the strong Coulomb coupling regime it is suitable to focus on the counterion surface properties. In the following sections we are going to study surface counterion distribution and diffusion.

\subsubsection{Counterion distribution}

Like in the ground state analysis, we characterize the counterion distribution via its surface correlation function. At non zero temperature, correlation functions are computed by averaging $\sum_{i \neq j} \delta\left(r^{\prime}-r_{i}\right) \delta\left(r^{\prime \prime}-r_{j}\right)$ over 1000 independent equilibrium configurations which are statistically uncorrelated. 
The results for monovalent counterions are depicted in Fig. 9(a) and Fig. 9(b) for $Z_{m}=60$ and $Z_{m}=180$ respectively. For both charges $Z_{m}$ the counterion distributions are weakly affected by charge discretization and $g_{c}^{(\mathrm{DCC})}(r)$ and $g_{c}^{(\mathrm{CC})}(r)$ are almost identical. A closer look on Fig. 9 reveals that the agreement between discrete and continuous distributions is even better for high macroion charge density $\left(Z_{m}=180\right)$ as expected. In fact for monovalent systems the pinning term $E_{\text {pin }}$ has its lowest magnitude so that, for sufficiently high $\sigma_{m}$, the fluctuating intra-dipole separation becomes comparable to the inter-dipole separation and discretization effects (i. e. pinning) are canceled. These pinning and unpinning aspects will be addressed in more details in the next section 4.1.2. As expected, the counterion positional order for discrete and continuous cases is much weaker at room temperature than in the ground state case (compare Fig. 9 and Fig. 2).

The results for multivalent counterions are depicted in Fig. 10. We now find that the counterion distributions are strongly affected by charge discretization, and especially the higher $Z_{c}$. This is in contrast with what was found in the ground state analysis Sec. 3.1.2 where no counterion motion occurs. This effect is of course due to the pinning (inhibition of large counterion motion) which is proportional to $Z_{c}$.

Note that all the statements above hold for the particular finite temperature $T_{0}$. However the effect of finite temperature discussed here should hold, at least qualitatively, for a large temperature range. For very low temperature one should recover all ground state properties mentioned in Sec. 3.1.

(a)

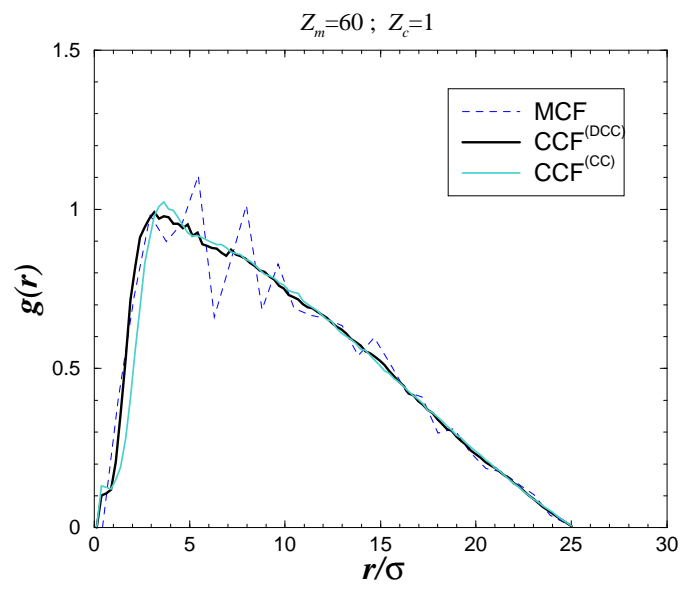

(b)

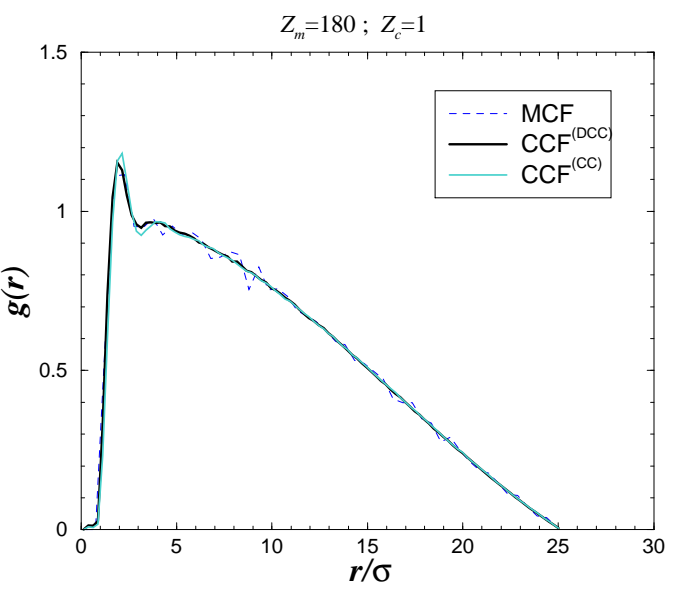

Fig. 9. Surface correlation functions at room temperature $T_{0}$ for monovalent counterions. The two counterion correlation functions (CCF) $g_{c}(r)$ are obtained for discrete colloidal charges (DCC) and for the central charge (CC): (a) $Z_{m}=60$ (b) $Z_{m}=180$. MCF stands for $g_{m}(r)$. 
(a)

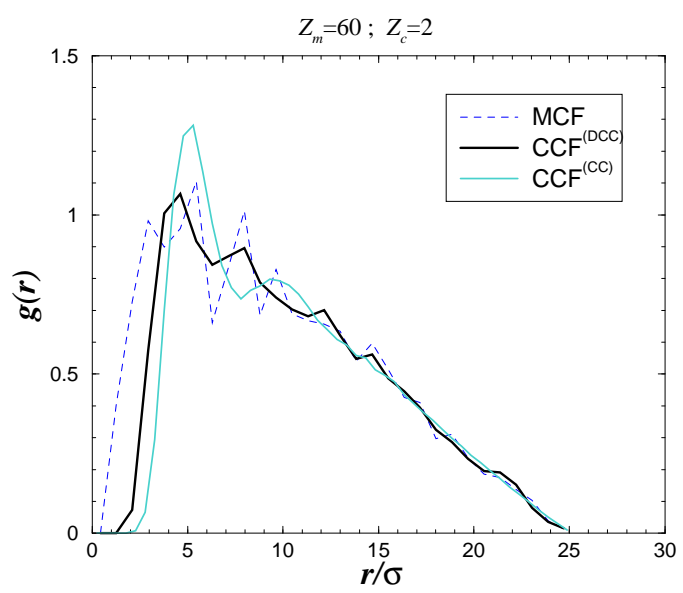

(c)

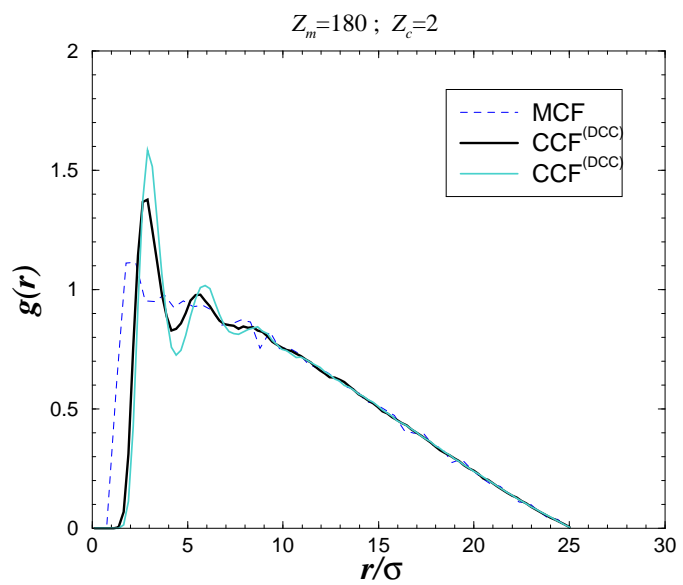

(b)

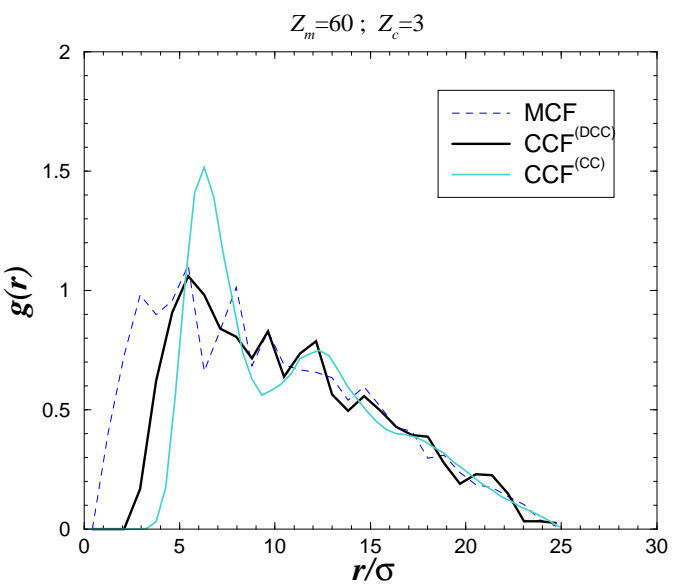

(d)

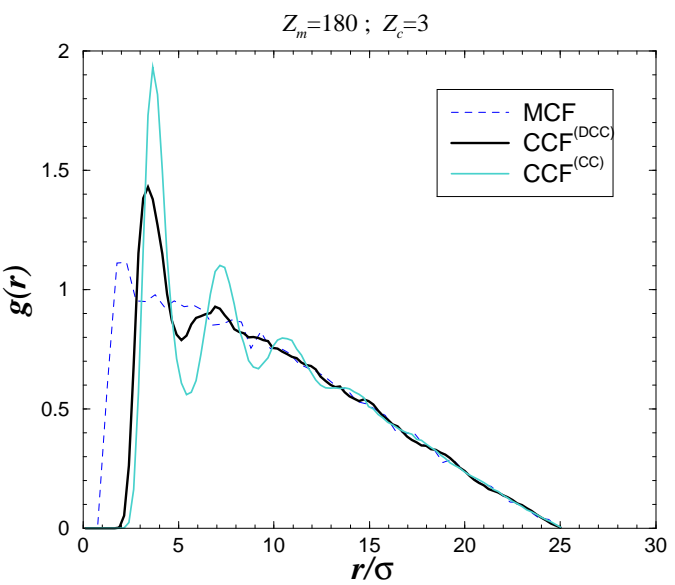

Fig. 10. Surface correlation functions at room temperature $T_{0}$ for multivalent counterions. The two counterion correlation functions $(\mathrm{CCF}) g_{c}(r)$ are obtained for discrete colloidal charges (DCC) and for the central charge (CC): (a) $Z_{m}=60, Z_{c}=2$ (b) $Z_{m}=60, Z_{c}=3$ (c) $Z_{m}=180, Z_{c}=2$ (d) $Z_{m}=180, Z_{c}=3$. MCF stands for $g_{m}(r)$.

\subsubsection{Surface diffusion}

This section is devoted to answer the following question: do the counterions only oscillate around the DCC sites or do they have also a large translational motion over the sphere?

To study this problem we introduce the following observable:

$$
\Delta x^{2}\left(t, t_{0}\right)=\frac{1}{t-t_{0}} \int_{t_{0}}^{t} d t^{\prime}\left[x\left(t^{\prime}\right)-x\left(t_{0}\right)\right]^{2}
$$

which is referred as the mean square displacement (MSD), where $x\left(t_{0}\right)$ repre- 
sents the position of a given counterion at time $t=t_{0}$ (at equilibrium) and $x\left(t, t_{0}\right)$ is its position at later time $t$. All particles lying within a distance $9.2 \sigma$ from the macroion center are radially projected on the macroion surface of radius $a=8 \sigma$ to give $x\left(t, t_{0}\right)$. The root mean square displacement (RMSD) $\Delta x\left(t, t_{0}\right)$ is defined as

$$
\Delta x\left(t, t_{0}\right)=\sqrt{\Delta x^{2}\left(t, t_{0}\right)} .
$$

Like for the surface correlation function, the RMSD is measured on the spherical surface (arc length) and it has a natural upper limit $\pi a$. For the case of free counterions (i. e. macroion central charge without pinning) the RMSD $\Delta x_{\text {free }}$ reads

$$
\Delta x_{\text {free }}=a \sqrt{\frac{\pi^{2}-4}{2}} \approx 13.7 \sigma .
$$

This quantity $\Delta x_{\text {free }}$ will be useful to refer to the "unpinned" state.

The results for discrete systems are sketched in Fig. 11 for $Z_{m}=60$ and $Z_{m}=180$. Monovalent counterions are free to move over the macroion surface for both bare charges $Z_{m}$ considered here. Moreover, our simulation data show that the counterions gradually become pinned with increasing $Z_{c}$. All these features are captured by the $Z_{c}$ dependency of the pinning term $E_{\text {pin }}$. For multivalent counterions, the degree of pinning increases with decreasing $Z_{m}$. This is due to the fact that the discrete charges get closer from each other by increasing $Z_{m}$ so that a counterion jump from site to site is energetically less demanding. For the continuous case, we have checked that for the same parameters counterions always have a large lateral motion and move all over the sphere.

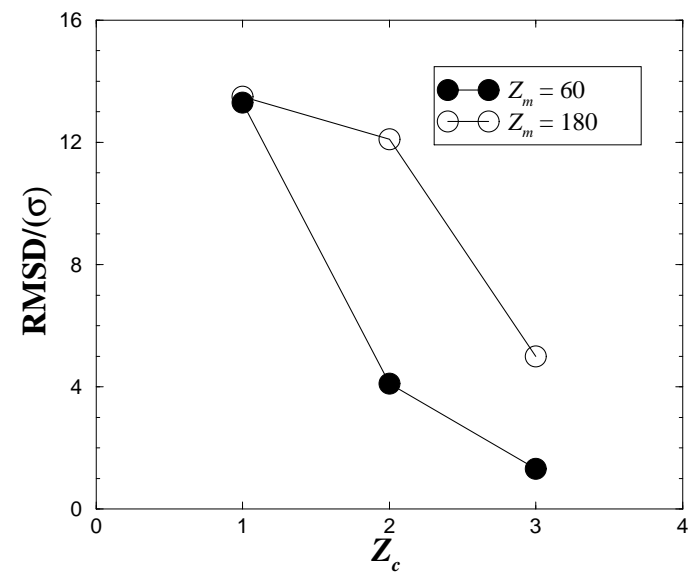

Fig. 11. Root mean square displacement (RMSD) as a function of counterion valence $Z_{c}$ for $Z_{m}=60$ and $Z_{m}=180$. Errors are smaller than symbols. 


\subsection{Moderate Coulomb coupling}

In this last part, the Bjerrum length corresponds to that of water at room temperature $\left(l_{B}=2 \sigma=7.14 \AA\right)$. For this moderate Coulomb coupling counterions occupy all the cell volume. Clearly, the probability of finding counterions plainly outside the macroion surface is no more negligible (in contrast with the strong Coulomb coupling). The target quantity is the fraction $P(r)$ of counterions lying within a distance $r$ from the macroion center and is defined as

$$
P(r)=N(r) / N_{c}
$$

with

$$
N(r)=\int_{r_{0}}^{r} 4 \pi r_{i}^{2} c_{v}\left(r_{i}\right) d r_{i}
$$

where $c_{v}(r)$ is the profile of the volume counterion concentration and $N(r)$ is the so-called integrated charge.

The results for $Z_{m}=60$ and $Z_{m}=12$ are sketched in Fig. 12(a) and Fig. 12 (b) respectively. For the highest charge, Fig. 12(a) shows that discretization effects are canceled for any counterion valence. On the other hand, for the small charge density case, Fig. 12(b) shows that discretization effects become important for multivalent counterions. In the present situation, the Coulomb coupling is five times less important than in the strong coupling case studied

(a)

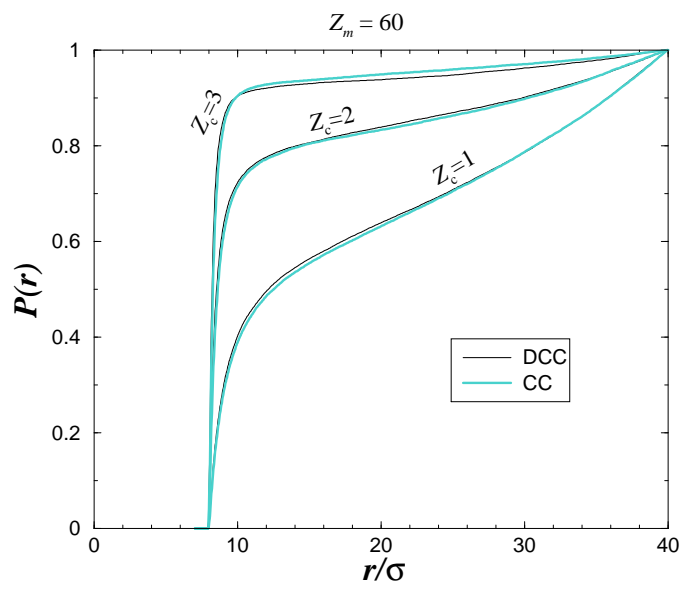

(b)

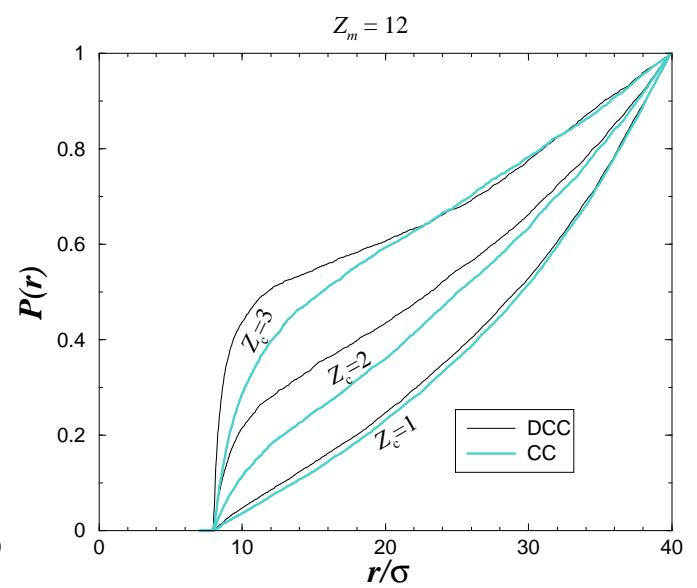

Fig. 12. Counterion fraction within a distance $r$ from the macroion center for different counterion valence $Z_{c}$. (a) $Z_{m}=60$ (b) $Z_{m}=12$. Data were obtained for discrete macroion charge distribution (DCC) and macroion central charge (CC). 
in Sec. 4.1. Therefore pinning effects can only be noticeable for sufficiently low $\sigma_{m}$ (here $Z_{m}=12$ ) and multivalent counterions.

\section{Conclusion}

We have performed MD simulations within the framework of the primitive model to study the coupled effects of macroion charge discretization and counterion valence. The macroion bare charge is carried by monovalent microions randomly distributed over the colloidal surface. Different correlational regimes were considered: (i) ground state and (ii) finite temperature.

Concerning the ground state analysis, we were interested in the counterion structure in the neutral state and the overcharging phenomenon. We demonstrated that the order in the surface counterion structure (disorder in counterion structure induced by the discrete random macroion charge distribution) is increased (decreased) by increasing macroion surface charge density $\sigma_{m}$ and/or counterion valence $Z_{c}$. For monovalent counterions, we showed that the ratio between the intra-ion pair (made up of a discrete colloidal surface ion and a counterion) distance and the mean distance between ion pairs is a fundamental quantity to describe counterion ordering. When overcharge comes into play similar effects occur. More precisely, for sufficiently high charge density $\sigma_{m}$ the overcharging with monovalent as well as multivalent counterions is quantitatively the same as the one obtained in the continuous case. For low $\sigma_{m}$, the overcharging with multivalent counterions can even be stronger in the discrete case than in the continuous case counterions. In contrast, for monovalent counterions overcharging is always weaker than in the continuous case but approaches the latter with increasing $\sigma_{m}$.

In the finite temperature case, strong and moderate Coulomb couplings were addressed. In the strong Coulomb coupling, we showed that counterion pinning is very weak for monovalent counterions but it increases with increasing $Z_{c}$ and decreasing $\sigma_{m}$. This involves an increasing disorder in the surface counterion structure with increasing $Z_{c}$ and decreasing $\sigma_{m}$. In the moderate Coulomb coupling corresponding to an aqueous situation, the volume counterion distribution is only affected for low $\sigma_{m}$ and multivalent counterions.

A future work will address the presence of added salts as well as the case of two interacting macroions. 


\section{Acknowledgements}

I thank C. Holm, A. Johner, K. Kremer and B. Shklovskii for helpful discussions. This work is supported by Laboratoires Européens Associés (LEA).

\section{References}

[1] J. Israelachvili, Intermolecular and Surface Forces, Academic, London, 1992.

[2] D. F. Evans and H. Wennerström, The Colloidal Domain, Wiley-VCH, New York, 1999.

[3] T. L. Hill, Statistical mechanics, Addison-Wesley, Reading, Mass., 1960.

[4] H. Wennerström, B. Jönsson and P. Linse, J. Chem. Phys. 76 (1982) 4665.

[5] V. Perel and B. Shklovskii, Physica 274A (1999) 446.

[6] B. Shklovskii, Phys. Rev. E 60 (1999) 5802.

[7] E. M. Mateescu, C. Jeppesen and P. Pincus, Europhys. Lett. 46 (1999) 493.

[8] J. F. Joanny, Europ. J. Phys. B 9 (1999) 117.

[9] E. Gurovitch and P. Sens, Phys. Rev. Lett. 82 (1999) 339.

[10] M. Lozada-Cassou, E. González-Tovar and W. Olivares, Phys. Rev. E 60 (1999) R17.

[11] M. Deserno, C. Holm and S. May, Macromolecules 33 (2000) 199.

[12] R. Messina, C. Holm and K. Kremer, Phys. Rev. Lett. 85 (2000) 872.

[13] R. Messina, C. Holm and K. Kremer, Europhys. Lett. 51 (2000) 461.

[14] T. T. Nguyen, A. Y. Grosberg and B. I. Shklovskii, Phys. Rev. Lett. 85 (2000) 1568.

[15] T. T. Nguyen, A. Y. Grosberg and B. I. Shklovskii, J. Chem. Phys. 113 (2000) 1110.

[16] R. Messina, C. Holm and K. Kremer, Eur. Phys. J. E 4 (2001) 363.

[17] R. Messina, C. Holm and K. Kremer, Phys. Rev. E 64 (2001) 021405.

[18] O. Spalla and L. Belloni, J. Chem. Phys. 95 (1991) 7689.

[19] S. Bhattacharjee, C. H. Ko and M. Elimelech, Langmuir 14 (1998) 3365.

[20] K. S. Schmitz, Langmuir 15 (1999) 2854.

[21] L. Bonsall and A. A. Maradudin, Phys. Rev. B 15 (1977) 1959.

[22] I. Rouzina and V. A. Bloomfield, J. Chem. Phys. 100 (1996) 9977. 